\title{
Thoracoscopic Correction of Post-traumatic Kyphosis with an Expandable Cage: Radiologic and Patient-Reported Outcomes
}

\author{
Arjen J. Smits, Jaap Deunk, Fred C. Bakker, Frank W. Bloemers \\ Department of Traumasurgery, VU University Medical Centre, Amsterdam, The Netherlands
}

\section{Study Design: Retrospective cohort study.}

Purpose: To determine the long-term functional and radiological outcomes after thoracoscopic post-traumatic kyphosis (PTK) correction (PTKC).

Overview of Literature: PTK after insufficient treatment of unstable fractures usually presents with pain and decreased back function. The results of PTKC using a minimally invasive thoracoscopic approach with an anterior titanium cage have not previously been reported.

Methods: Data in this study were collected from all patients who underwent thoracoscopic PTKC with an expandable cage between 2007 and 2017. Kyphosis and intervertebral body height were assessed on radiographic material. Quality of life (0OL) and functional outcome scores were determined by the Euroquol 5 dimensions (EO5D) and the Oswestry Disability Index (ODI). Additionally, satisfaction and subjective symptom improvement were determined.

Results: Fourteen patients were treated for symptomatic PTK using a combined thoracoscopic anterior and posterior approach. Nine patients received initial conservative fracture treatment and five patients underwent initial posterior fracture fixation. All patients presented with pain and without neurologic injury. The mean time between injury and PTKC was 15.4 months. Cobb angle (CA) improved with $10.6^{\circ}$ immediately after PTKC. During the first follow-up, $4.8^{\circ}$ kyphosis correction was lost, but CAs remained stable at longer follow-up. Bony fusion was achieved in $92 \%$ of the patients after 16 months. The majority of patients reported an improvement of symptoms 85 months after surgery, satisfaction with and willingness to undergo the procedure again. The mean EQ5D index score was 0.71 and the mean $\mathrm{ODI}$ score was 22.3 .

Conclusions: The results of minimally invasive thoracoscopic PTKC using an expandable cage were satisfactory. The majority of the patients were satisfied after treatment and no neurological complications occurred. Functional and QOL scores were fairly good. Whereas some postoperative kyphosis correction was lost over time, bony fusion was achieved in the majority of the patients. The thoracoscopic approach minimizes surgical morbidity, does not lead to serious complications, and provides a good option for PTKC.

Keywords: Post-traumatic; Kyphosis; Thoracoscopy; Spinal fractures; Cage; Anterior-posterior approach; Minimally invasive

Received Feb 26, 2019; Revised May 12, 2019; Accepted May 20, 2019

Corresponding author: Arjen J. Smits

Department of Traumasurgery, VU University Medical Centre, De Boelelaan 1117, room 7F020, 1081 HV Amsterdam, The Netherlands Tel: +31-204445234, Fax: +31-204445244, E-mail: aj.smits@vumc.nl, arjensmits@gmail.com 


\section{Introduction}

Undesirable late consequences of traumatic thoracolumbar fractures are spinal deformity and pain. This is mostly due to the loss of vertebral body height after insufficient conservative treatment of unstable fractures. However, this might also occur after a single posterior instrumentation as a result of insufficient load-sharing capacity [1], and non-union and/or osteonecrosis of the vertebral body with subsequent implant failure [2]. The post-traumatic deformity of the spine usually presents as severe kyphosis, which may cause pain and decreased back function [3-5]. Generally, two types of treatment options for post-traumatic kyphosis (PTK) correction (PTKC) are available: posterior osteotomies and anterior lengthening. Posterior osteotomies include pedicle subtraction osteotomies (PSO) and posterior column shortening (Smith-Peterson osteotomies). Anterior lengthening might be done using solely anterior fixation $[6,7]$ or with combined anterior and posterior fixation $[8,9]$.

While the correlation of kyphosis with functional outcomes has not been proven, it has been reported that kyphosis over $30^{\circ}$ leads to significant back pain $[4,5]$. Furthermore, patients with a preoperative sagittal index of more than $15^{\circ}$ have been reported in the study by Farcy et al. [10] as potentially benefiting from PTKC. In addition to pain, a new or increasing neurologic deficit is also an indication for the surgical treatment of PTK. Finally, the cosmetic appearance of a severe deformity can be a relative indication for surgical treatment. The applied surgical approach still mainly depends on the preference of the treating surgeon [11]. Posterior column shortening is especially effective in treating sagittal disbalance in degenerative multilevel deformities [12] and ankylosing spondylitis. However, most patients with a post-traumatic deformity do not have sagittal disbalance due to compensatory changes in other uninjured spine segments [4]. In these patients, the anterior lengthening on the level of the collapsed vertebral body can be considered as a logical alternative to PSO in treating PTK.

Augmentation of the anterior column with titanium cages instead of autografts prevents the disadvantages of autografts [13]. Additionally, the use of a minimally invasive thoracoscopic approach $[14,15]$ prevents the morbidity of an open technique $[16,17]$ and at the same time improves the visual exposure of the anterior column. The value of this approach has been proven in the treatment of unstable fresh spinal fractures [18]. The outcomes of anterior correction with an expandable cage using a minimal invasive thoracoscopic approach for PTKC, however, have not yet been reported. We therefore report the radiologic, clinical, and functional results of the first series of patients who have been treated through the abovementioned method for focal PTK.

\section{Materials and Methods}

All patients who underwent thoracoscopic PTKC between 2007 and 2017 in our level 1 trauma center (Amsterdam University Medical Center, Locatie VUmc, Amsterdam, the Netherlands) were analyzed. All patients had failed initial treatment of a traumatic thoracolumbar fracture. The initial treatment was conservative or surgical with posterior fixation, which was performed at a referring regional center or at our academic trauma center. The indications for PTKC were severe or increasing kyphosis with impairing pain.

\section{Data collection}

Baseline data and patient characteristics (age, sex, mechanism of injury, level and type of fractured vertebra, injury severity, neurologic injury, initial treatment, kyphosis correction date and method, implants, segments fused, and complications) were retrospectively collected from the hospital information system. Current quality of life (QOL) and back pain disability were assessed by two validated questionnaires, the Euroquol 5 dimensions (EQ5D) and the Oswestry Disability Index (ODI), respectively. The EQ5D was scored on five dimensions from which an index score was calculated based on the general population of a specific country with 1 corresponding to full health and 0 corresponding to death. Additionally, a Visual Analog Scale (VAS) score was filled in, corresponding to current health on the reported day $(100=$ full health and $0=$ worst health). The ODI consisted of 10 domains from which an overall low back pain disability score can be calculated $(100 \%=$ complete disability and $0 \%=$ no disability). A demographic questionnaire consisting of four questions concerning treatment satisfaction and numeric rating scale (NRS)-rated back pain over the last week was sent along with the validated questionnaires. The study was approved by the institutional medical ethical review board (METc VUmc) under case number 2017.183, and 


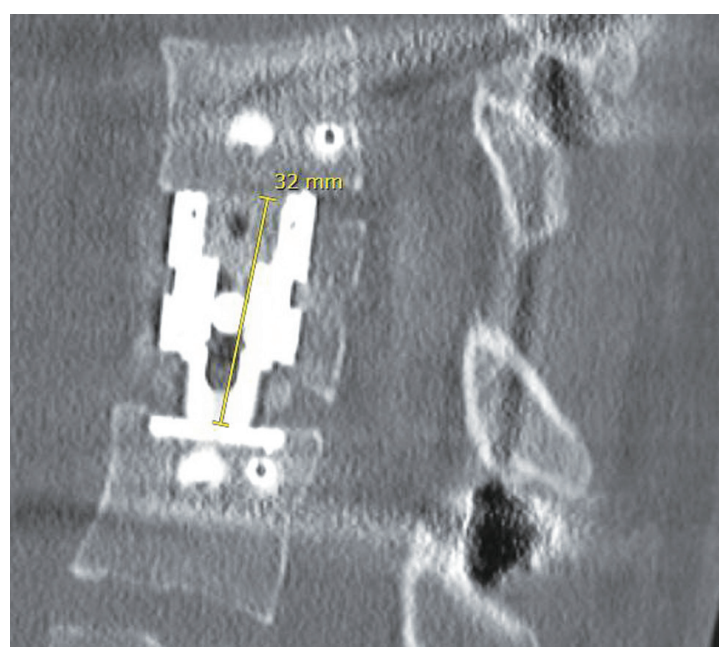

Fig. 1. Example of intervertebral body height measurement.

informed consent was obtained from all participants included in the study.

\section{Radiographic parameters}

All available radiographic materials were re-assessed by two separate authors for fracture characteristics (AO classification), kyphosis (Cobb angle [CA]), intervertebral body height (IBH), and bony fusion. Kyphosis, IBH, and bony fusion were assessed over time at each followup moment available. Kyphosis was assessed by CA on sagittal computed tomography (CT) when available or otherwise by magnetic resonance imaging or plain lateral radiographs [19]. Additionally, sagittal index was determined as segmental kyphosis (CA) adjusted for the baseline sagittal contour at that level $[4,10]$. Fusion was scored by two experienced spine surgeons on CT scan. IBH was identified as the distance between the center of the inferior and superior endplates of the vertebra above and below the index vertebra, measured on mid-sagittal CT (Fig. 1). If disagreement existed concerning the measurements or fracture classifications, another experienced spine surgeon was consulted.

\section{Surgical technique}

Two surgeons performed all surgeries. The PTKC procedure consisted of three stages in one session: thoracoscopic minimal invasive anterior release, posterior instrumentation and kyphosis correction, and thoracoscopic anterior fixation with a cage and anterolateral plating. The
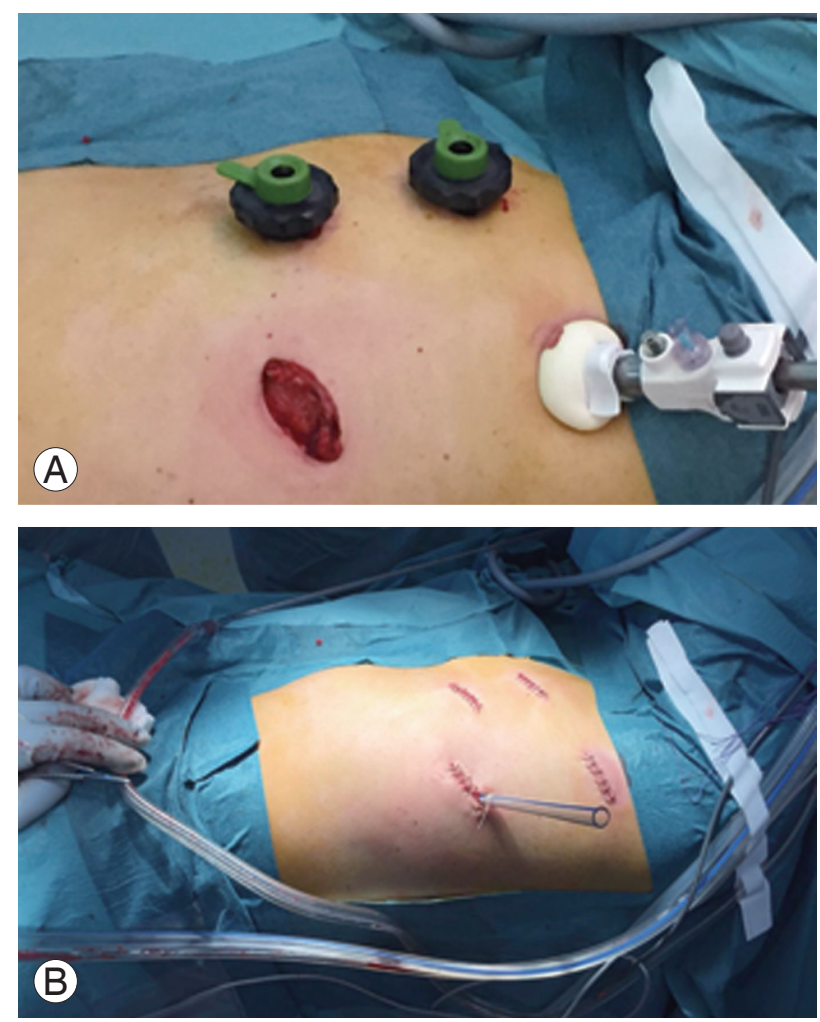

Fig. 2. (A) Preoperative situation with trocars in situ and (B) postoperative situation with closed portal incisions.

thoracoscopic procedure was performed as described by Beisse et al. [15] and Khoo et al. [18] and the cell saver was used during the procedure. In the first stage, the patient was positioned in the left or right lateral decubitus position depending on the level of the fractured vertebra and its relation to the anatomic position of the greater vessels. Single lung ventilation was then applied. The targeted vertebra was then identified under further fluoroscopic guidance and the portals were inserted into the thorax. The first portal was inserted under direct visualization using a slightly larger skin incision (approximately $4 \mathrm{~cm}$ ). The other trocars were inserted under direct visualization through the scope (Fig. 2A). The lung was retracted using the fan retractor, and, to access the caudal part of the thoracolumbar junction (T12-L2), the crus of the diaphragm was slightly detached and pushed caudally. To access the lumbar vertebrae, the psoas muscle was additionally partly dissected. Anterior release was performed by dissecting the anterior ligaments and ossifications, and a partial or complete corpectomy with discectomy was performed using a rongeur with maintenance of the posterior vertebral wall. Additionally, an anterior spinal cord decompression can be performed in this stage. Two lateral screws of 
Table 1. Patient characteristics

\begin{tabular}{|c|c|}
\hline Characteristic & Value \\
\hline No. of patients & 14 \\
\hline Male:female & $7: 7$ \\
\hline Age (yr) & $43 \pm 18$ \\
\hline Injury Severity Score & $12 \pm 9$ \\
\hline$<16(n=10)$ & $7.8 \pm 2.9$ \\
\hline$\geq 16(n=3)$ & $26.7 \pm 2.5$ \\
\hline Multiple fractures & $3(21)$ \\
\hline \multicolumn{2}{|l|}{ Fracture level } \\
\hline T6, T8, T9, T10, L2 & $1(7)$ \\
\hline $\mathrm{T} 7$ & $2(14)$ \\
\hline $\mathrm{T} 12$ & $4(29)$ \\
\hline L1 & $3(21)$ \\
\hline \multicolumn{2}{|l|}{ Fracture type $(\mathrm{AO})^{\mathrm{a})}$} \\
\hline A3 & $2(14)$ \\
\hline A4 & $12(86)$ \\
\hline B/C type & $1(7)$ \\
\hline \multicolumn{2}{|c|}{ Cobb angle before post-traumatic kyphosis } \\
\hline $10^{\circ}$ & $1(7)$ \\
\hline $20^{\circ}-30^{\circ}$ & $8(57)$ \\
\hline$>30^{\circ}$ & $5(36)$ \\
\hline \multicolumn{2}{|l|}{ Anterior segments fixation } \\
\hline 1 & $1(7)$ \\
\hline 2 & $12(86)$ \\
\hline 3 & $1(7)$ \\
\hline \multicolumn{2}{|l|}{ Posterior segments fixation } \\
\hline 2 & $7(50)$ \\
\hline 3 & $2(14)$ \\
\hline 4 & $2(14)$ \\
\hline 5 & $1(7)$ \\
\hline \multicolumn{2}{|l|}{ Cage type } \\
\hline Synex 2 & $2(14)$ \\
\hline Obelisc & $12(86)$ \\
\hline \multicolumn{2}{|c|}{ Additional bone substitute (to corpectomy) } \\
\hline Demineralized bone matrix & $9(64)$ \\
\hline Cerasorb & $2(14)$ \\
\hline Autologous crista bone & $2(14)$ \\
\hline Only corpectomy bone & $1(7)$ \\
\hline \multicolumn{2}{|l|}{ Cause of injury } \\
\hline Fall/jump from height & $5(36)$ \\
\hline Fall from stairs & $3(21)$ \\
\hline Traffic accident & $4(29)$ \\
\hline
\end{tabular}

(Continued to the next page)
Table 1. Continued

\begin{tabular}{lc} 
Characteristic & Value \\
\hline Fall & $1(7)$ \\
\hline Fall from horse & $1(7)$ \\
\hline
\end{tabular}

Values are presented as number, mean \pm standard deviation, or number $(\%)$, unless otherwise stated.

${ }^{\text {al }}$ Total amount of fractures overlaps total amount of patients because the B type fracture was associated with an A4 type fracture.

the anterolateral plating (MACS-TL; Braun, Melsungen, Germany) were already placed in each vertebra above and below the kyphotic vertebra. The corpectomy space was then temporarily filled up with a gauze and the wound was provisionally closed before continuing to the second stage. The patient was then positioned in the prone position. Pedicle screws and rods were inserted via an open approach and segmental kyphosis reduction was applied under fluoroscopic guidance by direct pressure and distractive forces on the pedicle screws. If the kyphosis was corrected sufficiently and fixated with the pedicle screws and rods, the wound was closed and the patient was again positioned in lateral decubitus position. The portals were then re-inserted, the gauze was removed from the corpectomy space, and the expandable titanium cage (Obelisc; Ulrich Medical, Ulm, Germany) was placed and expanded until it had a good grip on the endplates. The lateral plate (MACS-TL, Braun) was then placed over and fixated to the previously inserted lateral screws. Finally, the cage and anterolateral plate were surrounded with corpectomy bone augmented with a demineralized bone matrix ([Depuy Synthes, Raynham, MA, USA] or Cerasorb [Curasan, Kleinostheim, Germany]) to stimulate bony fusion. A collagen sponge coated with fibrinogen and thrombin was applied over the construct (Tachosil). The diaphragm and wounds were then closed and a thoracostomy tube was left in place for 48 hours (Fig. 2B). The patient was allowed to mobilize after 24 hours with physiotherapy.

\section{Statistics}

Continuous variables following a normal distribution were presented as mean \pm standard deviation (SD), and non-normally distributed variables were presented as median with interquartile range (IQR). Dichotomous or categorical variables were presented as proportions. A comparison of continuous variables with normal distri- 
bution between two groups was done by a $t$-test or linear regression. Correlations between continuous outcomes were tested visually for outliers with scatter plots and then assessed for significance with linear regression and if needed were corrected for outliers. Statistical significance was regarded as $p<0.05$. Statistical analyses were done using IBM SPSS ver. 22.0 (IBM Corp., Armonk, NY, USA).

\section{Results}

Between 2007 and 2017, a total of 14 patients were treated for symptomatic PTK using a thoracoscopically placed expandable cage. The mean age at injury was 42 years (range,

Table 2. Time from injury to PTKC

\begin{tabular}{lcc} 
& \multicolumn{2}{c}{ Weeks injury to PTKC } \\
\cline { 2 - 3 } Primary treatment & $\begin{array}{c}\text { Mean } \pm \text { standard } \\
\text { deviation }\end{array}$ & $\begin{array}{c}\text { Interquartile } \\
\text { range }\end{array}$ \\
\hline Pedicle screws and rods $(n=5)$ & $82 \pm 55$ & $15-156$ \\
Orthosis $(n=5)$ & $57 \pm 19$ & $24-68$ \\
$\begin{array}{l}\text { Non-operative, exact unclear } \\
(n=4)\end{array}$ & $55 \pm 58$ & $9-131$ \\
\hline
\end{tabular}

PTKC, post-traumatic kyphosis correction.
13-69 years), and 10 of these patients were referred from regional hospitals due to unsatisfying results of the primary treatment. All fractures were originally located between T6 and L2 and most were A4-type fractures. None of the patients had neurologic impairment before or after surgery. Additional patient characteristics are reported in Table 1. The mean time between injury and PTKC was $15.4 \pm 10.6$ months (range, 2-36 months) (Table 2). Fig. 3 shows an example of pre- and postoperative CT scans.

\section{Primary fracture treatment}

Primary fracture treatment consisted of posterior fixation with pedicle screws and rods in five patients. Nine patients were primarily treated with conservative treatment. Five of them received an orthosis. Two patients did not receive any initial treatment due to patient delay; in two referred patients, it remained unclear if any specific conservative treatment was received. Four of five patients treated by dorsal fixation as primary treatment underwent this treatment within 1 week after injury, and one patient was dorsally fixated after 10 weeks when conservative treatment appeared insufficient.
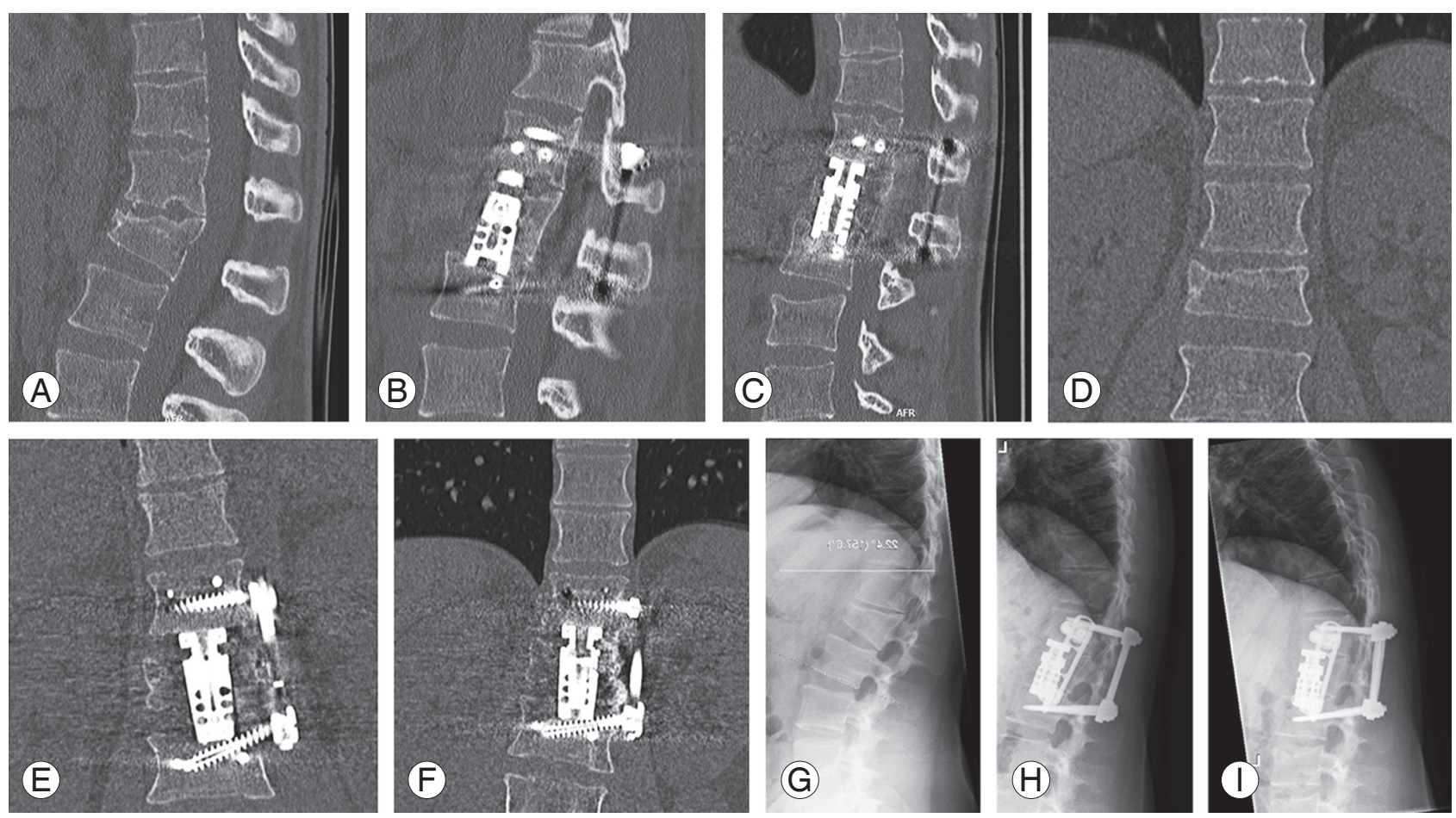

Fig. 3. Sagittal (A-C) and coronal computed tomography scan (D-F) and sagittal plain X-ray (G-I) of a patient treated with anterior-posterior fixation for PTK: preoperative (A, D, G); postoperative $(\mathbf{B}, \mathbf{E}, \mathbf{H})$; and 2 years postoperative $(\mathbf{C}, \mathbf{F}, \mathbf{I})$. 


\section{Indications and surgical technique}

All patients with PTK presented from injury with pain and severely progressed kyphosis over time. Ten patients were treated using a single-session three-staged anteriorposterior-anterior approach. Initially, nine cases were treated conservatively and one with posterior fixation, fusion, and secondary implant removal. This patient required a laminectomy due to initial posterior fusion and was therefore treated using the three-staged procedure. Posterior fixation is usually not performed with fusion in our center and this patient was initially treated in another country. Four patients underwent only anterior thoracoscopic treatment after failure of initial posterior instrumentation (without fusion). Two of these patients had already undergone removal of posterior instrumentation with secondary collapse. Blood loss for patients with only anterior instrumentation consisted of median $1,450 \mathrm{~mL}$
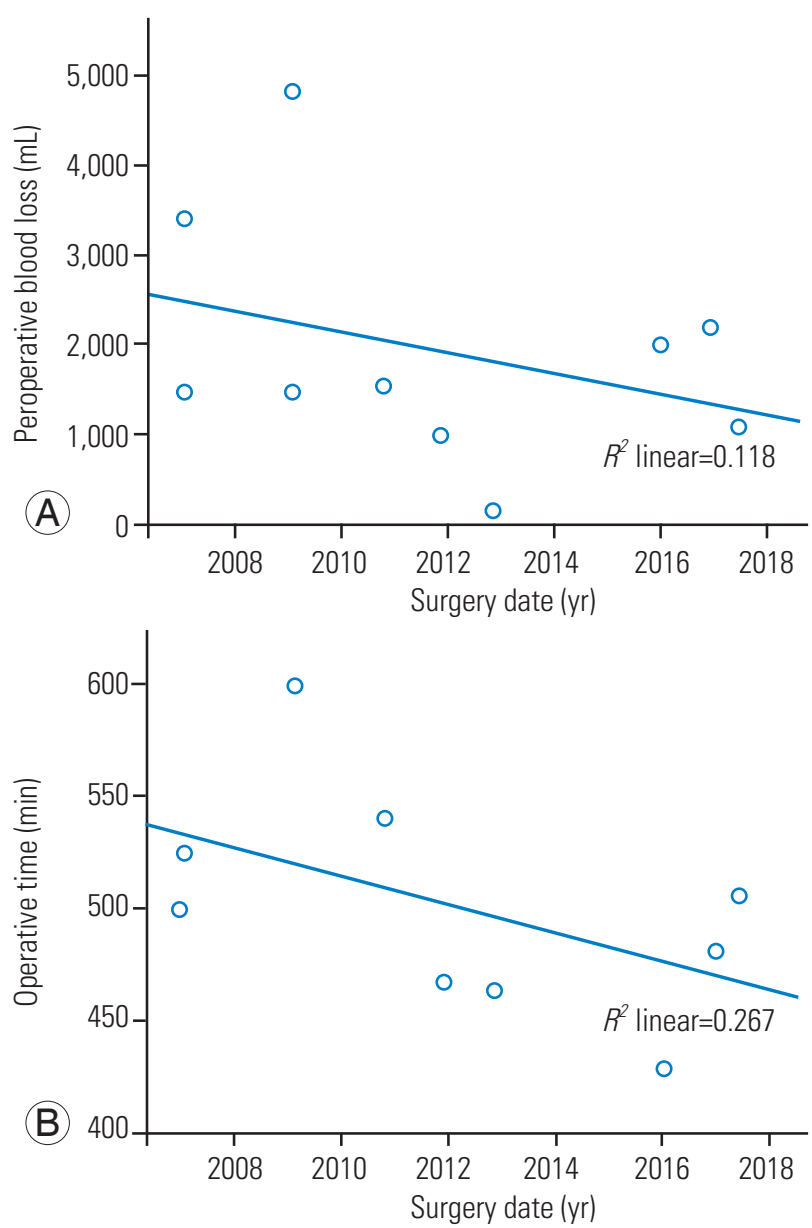

Fig. 4. Surgical blood loss (A) and operative time (B) evolution for the three-staged procedure $\left(\mathrm{n}=10 ; R^{2}=0.12 ; p=0.33\right)$. Corrected for one outlier $\left(\mathrm{n}=9 ; R^{2}=0.27 ; p=0.15\right)$.
(IQR, 1,225-1,975 mL); for the combined three-staged anterior-posterior-anterior approach, this was in total a median of 1,525 mL (IQR, 1,075-2,500 mL). The cell saver reduced blood loss to $1,032 \mathrm{~mL}$ (IQR, 174-1,320 $\mathrm{mL}$ ) and 1,375 mL (IQR, 813-2,457 mL), respectively. As experience with the technique increased over time, blood loss decreased, although not significantly $\left(R^{2}=0.12, p=0.33\right)$. The mean operative time was $286 \pm 49$ minutes for the anterior approach and $470 \pm 105$ minutes for the three-staged anterior-posterior-anterior approach. The operative time decreased over time, although not significantly $\left(R^{2}=0.27\right.$, $p=0.15$ ) (Fig. 4).

\section{Complications}

Two patients developed postoperative complications. One patient developed pulmonary embolism, superficial wound infection, and pleural empyema. These were successfully treated by anticoagulation, antibiotics, and chest tube drainage, respectively. Another patient developed pleural fluid without infection, which was successfully drained.

\section{Radiological outcomes}

All patients had available pre- and postoperative CT scans. The mean preoperative sagittal index was $24.7 \pm 6.6$ and the mean kyphosis (CA) was 26.1 \pm 9.0 . Kyphosis from preoperative to immediate postoperative $(n=14)$ improved significantly from $26.1^{\circ} \pm 9.0^{\circ}$ to $15.6^{\circ} \pm 7.4^{\circ}$ (mean difference $\left.[\mathrm{MD}] \pm \mathrm{SD}, 10.6^{\circ} \pm 8.4^{\circ} ; p<0.01\right)$. Over time, there was a minor but significant loss of initial kyphosis correction

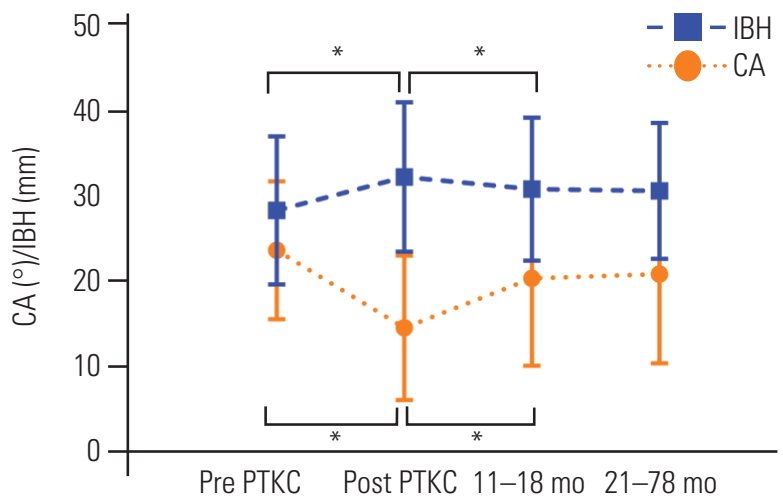

Fig. 5. CA and IBH evolution over time. Points display mean \pm standard deviation (bars). CA, Cobb angle; IBH, intervertebral body height; PTKC, post-traumatic kyphosis correction. ${ }^{*} p<0.05$; statistically significant difference (by paired $t$-test). 
$\left(\mathrm{MD} \pm \mathrm{SD}, 4.8^{\circ} \pm 4.0^{\circ} ; p<0.01\right)$. For seven patients, longterm radiographic follow-up was available, whereas the other patients, who had not yet reached this follow-up period, did their follow-up in another hospital, or were already discharged from follow-up. Patients with repetitive radiologic follow-up imaging available $(n=7)$ showed stable CAs over time from 11 to 78 months (Figs. 5, 6, Table 3). IBH ( $\mathrm{mm}$ ) increased significantly from pre- to

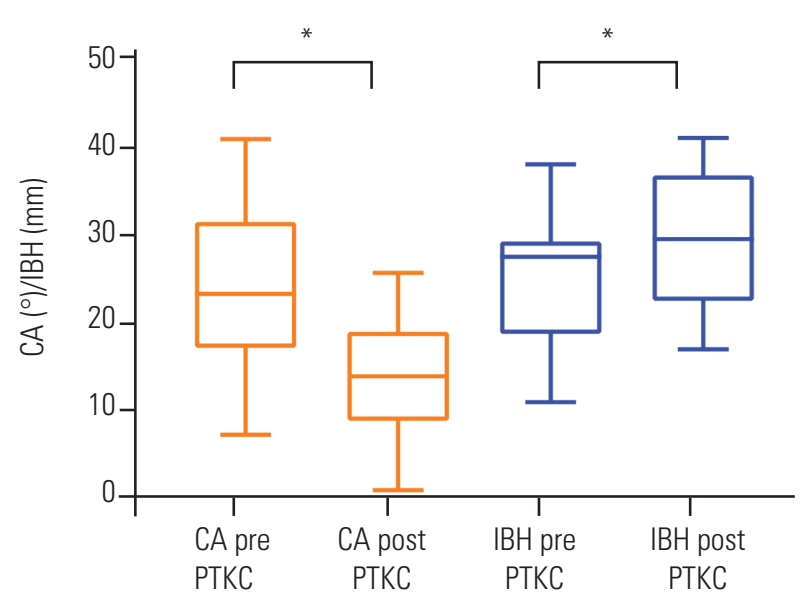

Fig. 6. $C A$ and $I B H$ before and after surgery. $C A$, Cobb angle; $I B H$, intervertebral body height; PTKC, post-traumatic kyphosis correction. ${ }^{*} p<0.05$; statistically significant difference (by paired $t$-test) as reported in Tables 3 and 4. postoperative ( $\mathrm{MD} \pm \mathrm{SD}, 4.4 \pm 2.8 ; p<0.01)$. At follow-up, IBH decreased but remained stable and was significantly larger compared to the preoperative value (Figs. 5, 6, Table 4). Fusion could be assessed on CT for 12 patients. Eleven (92\%) of these patients achieved bony fusion in $17.7 \pm 7.5$ months (mean $\pm \mathrm{SD})$. One had not achieved complete bony fusion after 36 months. The remaining two patients were followed up elsewhere.

\section{Functional outcomes and quality of life}

Of the 14 patients treated for PTK, nine patients had valid functional outcomes and QOL scores. Two patients were excluded from additional questionnaires due to psychiatric history and age below 16 at time of surgery, which was unlikely to produce representative answers. The followup period of one patient was too short ( $<6$ months) after surgery to provide reliable results. From the remaining 11 patients, a response rate of $82 \%$ was obtained. These nine patients filled in the questionnaires at $84.56 \pm 46.11$ months (mean \pm SD) after surgery (range, 7-125 months). The mean \pm SD ODI score was $22.3 \% \pm 18.5 \%$ (range, $0 \%-46 \%$ ) corresponding to moderate back pain-related disability. The mean \pm SD EQ5D index score was $0.71 \pm 0.23$ (range, 0.31-1.0) and the mean \pm SD EQ5D VAS score was

Table 3. Cobb angle at specific time points

\begin{tabular}{lcrrr} 
Moment 1 & Moment 2 & Cobb angle $\left({ }^{\circ}\right)$ & $p$-value & No. of patients \\
\hline Injury & Pre PTKC & $7.1 \pm 4.1$ & $0.002^{*}$ & 8 \\
\hline Pre PTKC & Post PTKC & $-10.6 \pm 8.4$ & $0.001^{*}$ & 14 \\
Post PTKC & $11-18$ mo & $4.8 \pm 3.8$ & $0.004^{*}$ & 11 \\
$11-18$ mo & $21-78$ mo & $0.5 \pm 1.1$ & 0.3 & 7 \\
Post PTKC & $21-78$ mo & $6.4 \pm 3.5$ & $0.005^{*}$ & 9 \\
\hline
\end{tabular}

Values are presented as mean difference \pm standard deviation, unless otherwise stated.

PTKC, post-traumatic kyphosis correction.

" $p<0.05$; statistical significant difference. ${ }^{\text {a }}$ By paired samples $t$-test.

Table 4. Intervertebral body height at specific time points

\begin{tabular}{lcccc} 
Moment 1 & Moment 2 & Intervertebral body height $(\mathrm{mm})$ & $p$-value ${ }^{\text {a) }}$ & No. of patients \\
Pre PTKC & Post PTKC & $4.4 \pm 2.8$ & $<0.001^{*}$ & 14 \\
Post PTKC & $11-18$ mo & $-1.5 \pm 1.1$ & $0.002^{*}$ & 11 \\
$11-18$ mo & $21-78$ mo & $-0.1 \pm 1.1$ & 0.7 & 7 \\
Post PTKC & $21-78$ mo & $-2.4 \pm 2.7$ & 0.04 & 8 \\
\hline
\end{tabular}

Values are presented as mean difference \pm standard deviation, unless otherwise stated.

PTKC, post-traumatic kyphosis correction.

${ }^{*} p<0.05$; statistical significant difference. ${ }^{a}$ By paired samples $t$-test. 


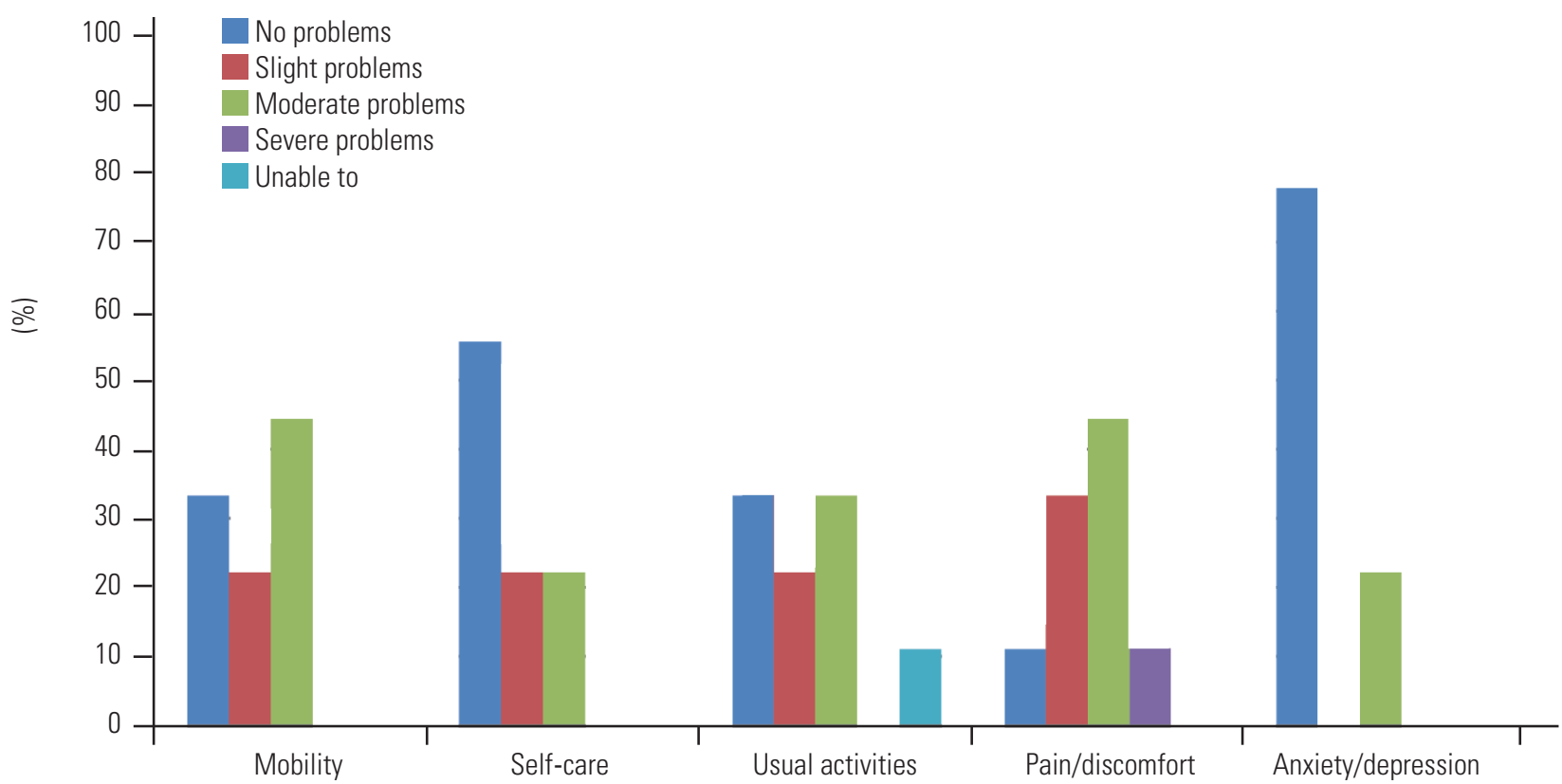

Fig. 7. EQ5D domain-specific outcomes. EQ5D, Euroquol 5 dimensions.

69.0 17.1 (range, 40-90). The domain-specific EQ5D scores are shown in Fig. 7.

Most patients were satisfied and had improvement of their symptoms. Most patients would undergo the procedure if they would hypothetically be in the same situation again, and there were no reports of severely increased symptoms (Fig. 8).

The mean \pm SD back pain NRS of the last week before the questionnaire was $4.11 \pm 2.32$ (range, 1-8). Analgesics used for back pain were reported by $33.3 \%(n=3)$ of the patients, and $66.7 \%$ of patients reported not using any back painrelated medication. One patient-reported using acetaminophen, one patient-reported using opiates, and one patientreported using both acetaminophen and opiates.

\section{Discussion}

PTK often occurs in the thoracolumbar and thoracic region. Due to unfamiliarity with the anterior approach, many surgeons tend to use PSOs with the need for long posterior instrumentation. The anterior approach, however, provides two-column stabilization with a short immobile segment, sparing remaining motion segments. Additionally, the approach enables the removal of any damaged intervertebral disc, which might have caused non-union due to collapse into the fracture. In an attempt to combine anterior fixation with minimal surgical morbidity, a mini-open approach was used $[9,20]$. An alterna-
Subjective effect of surgery on synptoms

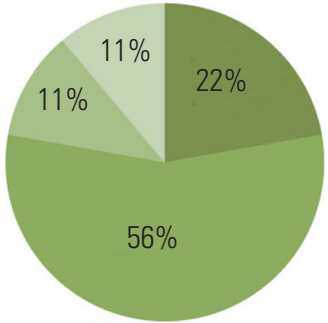

Satisfaction with surgery

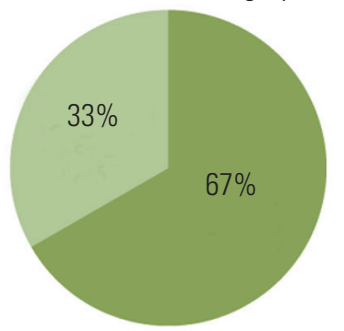

Satisfied

Not satisfied, nor dissatisfied
Would you undergo the procedure again if in the same situation?

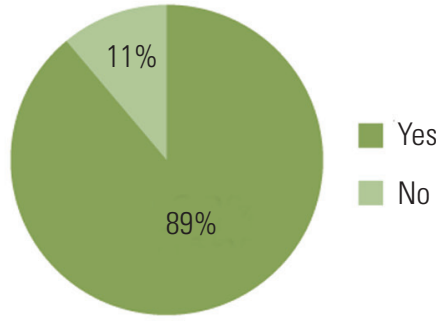

Fig. 8. Subjective patient outcomes.

tive is a posterolateral approach, but this affords limited exposure [21]. The thoracoscopic approach provides excellent exposure and two-column stability and minimizes 
surgical morbidity. This is the first study to describe the results and patient-reported outcomes after PTKC using a thoracoscopic approach.

PTK was corrected significantly during surgery, although some correction loss did occur over time. Most correction loss occurred in the first year, as reported previously, after a thoracoscopic anterior stabilization [16,22] and was partly due to some cage subsidence as the construct settled down. The majority of the patients reported improved symptoms, would undergo the surgery again, and were satisfied with surgery. Additional functional scores were comparable to those functional scores after PSO, and no neurologic complications or re-operations were reported.

\section{Radiologic parameters}

The average kyphosis correction was $10.6^{\circ}$, with a correction loss at final follow-up of $6.6^{\circ}$. This was comparable to the only other study that reported anterior-posterior treatment of patients with a preoperative $\mathrm{CA}$ of $<30^{\circ}$ [23]; however, in this study, bone grafts and an open approach were used. Other studies generally reported the results of patients with a preoperative CA of $>30^{\circ}[6-8]$. In these cases, the anterior-posterior approach corrects $30^{\circ}$ to $40^{\circ}$ kyphosis and PSO corrects even more $\left(35^{\circ}-47^{\circ}\right)$ with nearly any correction loss [24-27].

Patients with a preoperative sagittal index of $>15^{\circ}$ have been reported by Farcy et al. [10] to potentially benefit from PTKC. All patients presented with pain and had a sagittal index of $>15^{\circ}$, which was not always accompanied by extreme CAs. This strengthens the idea that the sagittal index $\left(>15^{\circ}\right)$ is a better indication for PTK surgery than $\mathrm{CA}$ alone as the sagittal index corrects for the physiologic difference in CA of different spine regions.

\section{Clinical outcome}

Although preoperative kyphosis generally did not exceed $30^{\circ}$, the mean sagittal index was largely more than $15^{\circ}$ and all patients presented with symptoms of severe pain. Symptoms improved after surgery in most patients, even while some correction loss did occur over time. The majority $(>75 \%)$ of the patients benefited from surgery by symptoms being largely or completely improved after surgery. The average back pain-related NRS score for the last week was 4.11 and only three patients still used back pain-related analgesics. Successful pain decrease was also reported after anterior PTKC in patients with severe kyphosis $\left(>30^{\circ}\right)$ treated with bone grafts, cages, and plating $[6,7,21]$. Furthermore, $67 \%$ of the patients reported to be satisfied with the procedure and there were no patients who were dissatisfied. Additionally, $89 \%$ of patients reported to be willing to undergo the procedure again if in the same situation, which was a higher percentage compared to other studies that used a conventional anterior approach $[7,28]$. This further indicates the effectiveness of the procedure on patient-reported outcomes. For the treatment of PTK with PSO, large pain decreases were also reported [24-26], although there was no further reporting of subjective results.

\section{Oswestry Disability Index and Euroquol 5 dimensions}

Functional scores after the thoracoscopic anterior treatment of PTK are acceptable and comparable to those treated using a posterior approach. The average reported ODI score in this study (22.3) corresponded to moderate back pain disability, which was comparable to most studies that reported the results of PTK treated using a modified PSO $[25,27,29]$. Although one study reported a better ODI after PSO [24], worse scores were also reported after PSO for deformities [30]. A previous comparison of a posterior-anterior approach showed an increased improvement in ODI scores after PSO, although the anterior procedure was not thoracoscopic and applied only a plate instead of a cage [28].

The mean EQ5D index score reported in this study was 0.71. QOL after PTK treated with the use of an anterior approach had not yet been reported. Our patients, however, scored considerably better compared to patients treated with PSO for adult deformities including PTK (0.56) [30].

\section{Surgical parameters and complications}

The anterior-posterior approach took considerably longer compared to the anterior-only approach, 470 versus 286 minutes, respectively, which was largely due to the repositioning of the patient. The reported surgical duration for PSO ranged from 149 to 275 minutes [24-30]. The thoracoscopic anterior-only approach was slightly longer; however, we only reported data from four patients. The procedure was performed by experienced trauma surgeons with expertise in spinal trauma. Apart from tho- 
racoscopic trauma-related spine surgery and acute (intra) thoracic injuries, these surgeons do not perform other thoracoscopic procedures on a routine basis. They are, however, also trained as general surgeons and have abundant experience with laparoscopic surgery. Although the surgeons had experience with the anterior approach, over time a decrease in operative time was shown for the threestaged procedure. The presence of a learning curve might be explained by the technically demanding technique and the relative infrequent application of PTKC.

The blood loss in the anterior-only approach $(1,450$ $\mathrm{mL}$ ) was mainly a result of continuous oozing from the vertebral body during corpectomy. For the conventional anterior approach, this ranged from 470 to $1,250 \mathrm{~mL}[6,8]$; for the posterior approach, this ranged from 643 to 2,300 $\mathrm{mL}$ [24-30]. The difference in blood loss might be due to individual patient characteristics of the described patients and emphasizes the importance of using a cell saver during the procedure. This substantially reduced the net blood loss to amounts that were within the range reported in the literature. Furthermore, just as for operative time, a decrease in blood loss over time was shown, possibly due to a learning curve. Additionally, it might be emphasized that the technique does not primarily aim to reduce blood loss but merely prevents thoracotomy-related complications such as pain and might lead to good technical results due to improved vision.

Two patients developed complications, one minor and one major. Pleural fluid is a known complication of the thoracoscopic approach and can often be treated without residual symptoms. The thoracoscopic approach is applied to minimize surgical damage and associated complications such as post-thoracotomy syndrome [16,21]. Compared to previous studies with the anterior open approach for PTKC $[6,21,23]$, the number of complications in our group was low. Furthermore, no neurologic complications occurred, which were reported after PSOs [24,26,27].

\section{Strengths and limitations}

The retrospective design and small patient sample are obvious limitations of this study. Although no validated preoperative results were available, current validated functional and QOL scores were obtained. No such results have yet been reported in the literature for this procedure. The long-term radiologic follow-up periods were heterogeneous due to some patients (mostly referrals) being followed up in other hospitals. Additionally, the followup periods of patient-reported outcome measures were heterogeneous because of the large spread in surgery dates and the cross-sectional nature of the questionnaires. Due to the small patient sample, maximal efforts were made to collect responses to the questionnaires, which resulted in a high response rate of $82 \%$.

\section{Conclusions}

The results of the thoracoscopic minimally invasive treatment of established PTK were satisfactory. Functional scores were good, comparable to other treatments for PTK and with no neurologic complications. Although some postoperative kyphosis correction is lost over time, bony fusion was achieved in the majority of the patients. The majority of the patients who completed the questionnaires reported improvement of symptoms and were satisfied and willing to undergo the procedure again. Although larger studies are warranted, the thoracoscopic approach seems safe, technically sound, and a good option in treating PTK.

\section{Conflict of Interest}

No potential conflict of interest relevant to this article was reported.

\section{Acknowledgments}

This research received no specific grant from any funding agency in the public, commercial, or not-for-profit sectors. This work represents original work and has not been published or presented before.

\section{ORCID}

Arjen J. Smits: https://orcid.org/0000-0001-7630-8333

Jaap Deunk: https://orcid.org/0000-0002-7749-3849

Frank W. Bloemers: https://orcid.org/0000-0002-8393-4946

\section{Author Contributions}

All authors declare that they have met the criteria for inclusion in the authors' list; they have made substantial contributions to the concept and design of the study, data analysis, revision of the article for intellectual content. 
They believe it represents honest work and have all fully read and approved the final version to be published.

\section{References}

1. McCormack T, Karaikovic E, Gaines RW. The load sharing classification of spine fractures. Spine (Phila Pa 1976) 1994;19:1741-4.

2. Alanay A, Acaroglu E, Yazici M, Oznur A, Surat A. Short-segment pedicle instrumentation of thoracolumbar burst fractures: does transpedicular intracorporeal grafting prevent early failure? Spine (Phila $\mathrm{Pa}$ 1976) 2001;26:213-7.

3. Schulz R, Melcher RP, Garib MC, Schulz H, Weissman K, Harms J. Does kyphotic deformity correlate with functional outcomes in fractures at the thoracolumbar junction treated by $360^{\circ}$ instrumented fusion? Eur J Orthop Surg Traumatol 2014;24 Suppl 1:S93-101.

4. Buchowski JM, Kuhns CA, Bridwell KH, Lenke LG. Surgical management of posttraumatic thoracolumbar kyphosis. Spine J 2008;8:666-77.

5. Gertzbein SD. Scoliosis Research Society: multicenter spine fracture study. Spine (Phila Pa 1976) 1992;17:528-40.

6. Wang Q, Xiu P, Zhong D, Wang G, Wang S. Simultaneous posterior and anterior approaches with posterior vertebral wall preserved for rigid post-traumatic kyphosis in thoracolumbar spine. Spine (Phila $\mathrm{Pa}$ 1976) 2012;37:E1085-91.

7. Benli IT, Kaya A, Uruç V, Akalin S. Minimum 5-year follow-up surgical results of post-traumatic thoracic and lumbar kyphosis treated with anterior instrumentation: comparison of anterior plate and dual rod systems. Spine (Phila Pa 1976) 2007;32:986-94.

8. Lee JH, Oh HS, Choi JG. Comparison of the posterior vertebral column resection with the expandable cage versus the nonexpandable cage in thoracolumbar angular kyphosis. Clin Spine Surg 2017;30:E398406.

9. Marre B. Management of posttraumatic kyphosis: surgical technique to facilitate a combined approach. Injury 2005;36 Suppl 2:B73-81.

10. Farcy JP, Weidenbaum M, Glassman SD. Sagittal index in management of thoracolumbar burst fractures. Spine (Phila Pa 1976) 1990;15:958-65.

11. Schoenfeld AJ, Wood KB, Fisher CF, et al. Post- traumatic kyphosis: current state of diagnosis and treatment: results of a multinational survey of spine trauma surgeons. J Spinal Disord Tech 2010;23:e1-8.

12. Cecchinato R, Berjano P, Damilano M, Lamartina C. Spinal osteotomies to treat post-traumatic thoracolumbar deformity. Eur J Orthop Surg Traumatol 2014;24 Suppl 1:S31-7.

13. Briem D, Rueger JM, Linhart W. Osseous integration of autogenous bone grafts following combined dorsoventral instrumentation of unstable thoracolumbar spine fractures. Unfallchirurg 2003;106:195-203.

14. Noor A, Bloemers FW, Bakker FC. Surgical correction of post-traumatic kyphosis: a thoracoscopic approach. Ned Tijdschr Geneeskd 2013;157:A5427.

15. Beisse R. Endoscopic surgery on the thoracolumbar junction of the spine. Eur Spine J 2006;15:687-704.

16. Schnake KJ, Stavridis SI, Kandziora F. Five-year clinical and radiological results of combined anteroposterior stabilization of thoracolumbar fractures. J Neurosurg Spine 2014;20:497-504.

17. Oskouian RJ Jr, Johnson JP. Vascular complications in anterior thoracolumbar spinal reconstruction. J Neurosurg 2002;96(1 Suppl):1-5.

18. Khoo LT, Beisse R, Potulski M. Thoracoscopicassisted treatment of thoracic and lumbar fractures: a series of 371 consecutive cases. Neurosurgery 2002;51(5 Suppl):S104-17.

19. Kuklo TR, Polly DW, Owens BD, Zeidman SM, Chang AS, Klemme WR. Measurement of thoracic and lumbar fracture kyphosis: evaluation of intraobserver, interobserver, and technique variability. Spine (Phila Pa 1976) 2001;26:61-6.

20. Berjano P, Damilano M, Lamartina C. Sagittal alignment correction and reconstruction of lumbar posttraumatic kyphosis via MIS lateral approach. Eur Spine J 2012;21:2718-20.

21. Kostuik JP, Matsusaki H. Anterior stabilization, instrumentation, and decompression for post-traumatic kyphosis. Spine (Phila Pa 1976) 1989;14:379-86.

22. Graillon T, Farah K, Rakotozanany P, et al. Anterior approach with expandable cage implantation in management of unstable thoracolumbar fractures: results of a series of 93 patients. Neurochirurgie 2016;62:7885.

23. Been HD, Poolman RW, Ubags LH. Clinical outcome and radiographic results after surgical treatment of post-traumatic thoracolumbar kyphosis following 
simple type A fractures. Eur Spine J 2004;13:101-7.

24. Gao R, Wu J, Yuan W, Yang C, Pan F, Zhou X. Modified partial pedicle subtraction osteotomy for the correction of post-traumatic thoracolumbar kyphosis. Spine J 2015;15:2009-15.

25. Hu W, Wang B, Run H, Zhang X, Wang Y. Pedicle subtraction osteotomy and disc resection with cage placement in post-traumatic thoracolumbar kyphosis, a retrospective study. J Orthop Surg Res 2016;11:112.

26. Xi YM, Pan M, Wang ZJ, et al. Correction of posttraumatic thoracolumbar kyphosis using pedicle subtraction osteotomy. Eur J Orthop Surg Traumatol 2013;23 Suppl 1:S59-66.

27. Zhang X, Zhang X, Zhang Y, Wang Z, Wang Y. Modified posterior closing wedge osteotomy for the treatment of posttraumatic thoracolumbar kyphosis. J
Trauma 2011;71:209-16.

28. El-Sharkawi MM, Koptan WM, El-Miligui YH, Said GZ. Comparison between pedicle subtraction osteotomy and anterior corpectomy and plating for correcting post-traumatic kyphosis: a multicenter study. Eur Spine J 2011;20:1434-40.

29. Jo DJ, Kim YS, Kim SM, Kim KT, Seo EM. Clinical and radiological outcomes of modified posterior closing wedge osteotomy for the treatment of posttraumatic thoracolumbar kyphosis. J Neurosurg Spine 2015;23:510-7.

30. Eskilsson K, Sharma D, Johansson C, Hedlund R. Pedicle subtraction osteotomy: a comprehensive analysis in 104 patients: does the cause of deformity influence the outcome? J Neurosurg Spine 2017;27:56-62. 